\title{
Effects of bilateral alternation on perceived temporal uniformity of auditory and somesthetic pulse trains
}

\author{
MICHAEL A. NAKAO and SEYMOUR AXELROD \\ Department of Psychiatry, State University of New York at Buffalo, Buffalo, New York 14214
}

\begin{abstract}
Certain attempts to account for the apparent loss of information when stimuli are presented in rapid alternation between the two sides of the body imply that some elements of the alternatingstimulus train are deleted. This implication forces the prediction that the train of surviving stimuli will be nonuniformly spaced. Yet observers do not report such temporal nonuniformity. The perceptibility of actual nonuniformity in stimulus trains was here found to be strikingly poorer for bilaterally alternating than for unilateral pulses, a finding qualitatively consonant with deletion models. Quantitatively, however, thresholds for perceived nonuniformity were so low as to require that deletion models include a mechanism allowing central respacing of the surviving train.

"Stream segregation" is considered as an alternative to deletion models.
\end{abstract}

Brief auditory or somesthetic stimuli presented in regular rapid alternation to the two ears or the two sides of the body are less efficiently processed than stimuli presented unilaterally (Axelrod, Guzy, \& Diamond, 1968; Axelrod \& Nakao, 1974; Harvey \& Treisman, 1973; Holden, 1973), or simultaneously to the two sides (Treisman, 1971). Two classes of attempts to explain the inefficiency have invoked (1) contralateral neural inhibition (Axelrod et al., 1968; Axelrod \& Nakao, 1974; Harvey \& Treisman, 1973), and (2) an inability of attention to keep pace with the incoming alternating stimuli because of the time required to "reset the parameters" after each stimulus (Treisman, 1971) or to perform orientational (motor) shifts supposed to accompany or underlie the attention-shifting (Kahneman, 1970; Guzy \& Axelrod, 1972).

Although conceptually disparate, these explanations share an implicit assumption that, at some stage of processing, some of the stimulus pulses will be deleted and be lost to subsequent stages. They also demand that the train consisting of the survivors be temporally nonuniform, on the assumption that fewer than $50 \%$ of the stimuli are deleted. (This

This study was supported by a research grant (No. 06862) from the National Institute of Neurological Diseases and Stroke, by General Research Support Grant RR05400, and by a fellowship to M. A. Nakao from the United Way of Buffalo and Erie County. The results were reported at the meetings of the Eastern Psychological Association, Philadelphia, April 1974. Thanks are due to Michael L. Steinfeld, Roger J. Wilson, and Edward Nash for technical assistance; to Richard Srebro and Michael Behbehani for generous help in computer simulations of the lateral-inhibition model; and to Lillian Leiber and David S. Emmerich for helpful comments on earlier versions. Requests for reprints should be sent to Seymour Axelrod, Department of Psychiatry, SUNYAB, 2211 Main Street, Buffalo, New York 14214. assumption will be justified below.) Detailed illustrations will help make clear how deletion follows from the underlying models.

The first illustration is based on a lateral-inhibition account, whose central idea is that the activity of a neural structure, $A$, inhibits the activity of structure $B$, the degree of inhibition depending on the magnitude of response of structure $A$. The inhibition decays exponentially over time, and if the inhibition is reciprocal, then B's activity also inhibits the response of $\mathbf{A}$.

Consider the case of repetitive, alternating, and equal stimulation of auditory structures left and right, each of which responds to a single isolated click with magnitude $R_{1}$. Following Walley and Weiden (1973), ignore the threshold for inhibition, and assume no self-inhibition for either structure. The response of the structure responsive to the $i^{\text {th }}$ stimulus will be given by

$\mathbf{R}_{\mathrm{i}}=\mathbf{R}_{\mathbf{l}}-\mathbf{K}^{\prime}\left(\mathrm{W}_{1} \mathbf{R}_{\mathbf{i}-1}+\mathrm{W}_{2} \mathbf{R}_{\mathbf{i}-3}+\mathrm{W}_{3} \mathbf{R}_{\mathbf{i}-5} \ldots\right)$

where $K^{\prime}$ is a scaling factor and the Ws are a set of exponentially decreasing weighting coefficients. The magnitude of the $i^{\text {th }}$ response is thus equal to $R_{1}$, decremented by an inhibitory term proportional to the sum of the residual inhibitions accumulated over some number of previous responses of the contralateral structure.

In the example presented in Figure 1, $R$ is plotted as a function of click number; $R$ was set equal to unity, $\mathrm{K}^{\prime}=.21$, and the inhibition was assumed to summate over the last 10 alternate iterations (responses of the opposite-ear structure), beginning with the immediately preceding one. The weights 


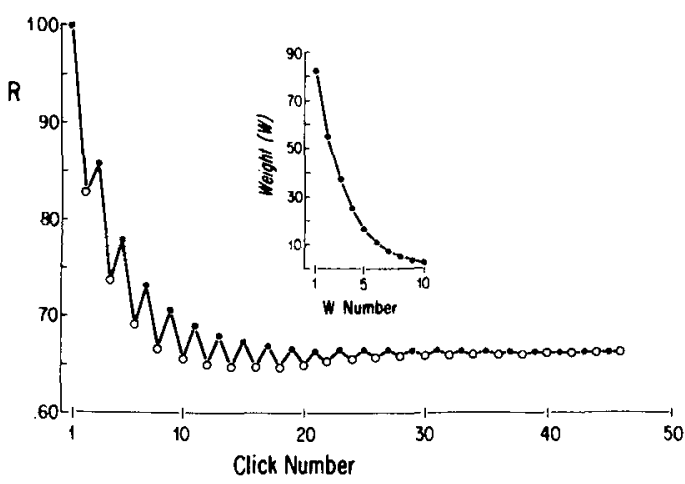

Figure 1. Magnitudes of response to alternating clicks (filled circles, left ear; open circles, right ear), as a function of ordinal position of the click, derived from a model based on reciprocal lateral inhibition (Equation 1), with $K^{\prime}=.21$; the inset shows the weighting function.

used (inset) are solutions of $W=e^{-2 x}, X$ taking the 10 successive values $1,3,5, \ldots 19$.

It is reasonable to assume that the probability, $\mathrm{Pr}$, that a click will be processed will be some direct function of the R it generates; for simplicity's sake, assume that $\mathrm{Pr}=\mathrm{R}$. In Figure 1 , filled circles represent the left responses, and open circles, the right responses. For any pair of consecutive responses, the difference between them divided by their mean was kept $\leqslant .2$. If differences of this order are assumed to be perceptually negligible-i.e., if the preasymptotic oscillation between filled and open circles in Figure 1 is ignored-then neither ear will be more likely than the other to have its clicks processed. In this probabilistic situation, the fate of individual clicks is not specified, and the result would be a train of randomly spaced survivors with a steady state processing probability of .66, the asymptotic value. The corresponding loss is $34 \%$.

Our second illustration-again, using arbitrarily chosen values-derives from an attention- (or orientation-) shifting model (Figure 2). Alternating stimuli are presented every $125 \mathrm{msec}(8 / \mathrm{sec})$, but the subject requires $178 \mathrm{msec}$ of dead transit time to shift his attention. Assume (a) that the subject can process a click only if his attention is at the stimulated ear at the moment of stimulation, or within some brief period thereafter during which the trace of the click remains retrievable; (b) that what cues the subject to shift his attention is the detection of a click; and (c) finally, that for simple stimuli of this type, attention can be divided between the ears before the onset of the train, so that processing of the first click can begin almost simultaneously with its presentation.

The first (left-ear) click appears (vertical line) and is detected. Attention is then shifted to the right ear, arriving after the second click, but within the trace period (horizontal bar), so that Click 2 is processed. Click 3 is likewise detected and processed, and so is Click 4, attention having arrived just within the retrievability period. Attention is then shifted to the left ear, but too late to detect Click 5 . Because the cue to shift has not occurred, the subject's attention remains at the left ear for the time d, so that Click 6 is also missed. Click 7 then appears and is processed. Attention shifts to the right ear, and the sequence is repeated: 4 clicks processed, 2 deleted, 4 processed, 2 deleted, and so on. The result is loss of one third of the presented clicks, the survivors constituting a dichotically alternating train with interclick intervals (milliseconds) of 178 , $178,178,178+d, 178,178,178,178+d$, and so on.

The foregoing examples were deliberately rigged to generate (a) deletions of about $30 \%$, and (b) trains of survivors consisting of both left and right clicks, because of the context provided by previous experiments from this laboratory: (a) An input train consisting of alternating left and right clicks at $\mathbf{8 - 4 0}$ clicks/sec is typically perceived as about $60 \%-70 \%$ as fast as an otherwise identical train in which all the clicks go to one ear (Axelrod, et al., 1968); i.e., about $30 \%-40 \%$ of the alternating clicks appear to be "lost." A somewhat lower estimate of the amount of loss derives from a study by Guzy and Axelrod (1972), who found the apparent numerosity of alternating trains of 20 clicks presented at $8 / \mathrm{sec}$ to be $23 \%$ less than that of monotic clicks at the same rate. ${ }^{1.2}$ (b) The alternating train is correctly perceived as constituted of clicks to both ears.

It is important to note that if deletion of the order of $20 \%-40 \%$ is indeed occurring, just the mathematics of the situation demands that the train consisting of the survivors cannot be temporally uniform. ("Temporally uniform" is used here to describe a

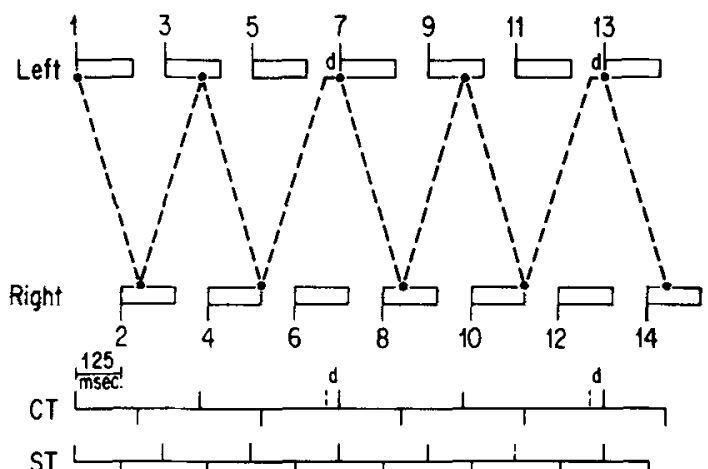

Figure 2. The operation of an attention- or orientation-shifting model, with minimum transit time longer than the interstimulus interval. The stimulus train consists of numbered clicks (vertical lines), each followed by a trace. In this example, alternating stimuli are presented every 125 msec, but the subject requires 178 msec to shift his attention (dashed line). The trace lasts at least 160 msec. Perception of a click, and the cue to shift, occur at the dots. The delay between arrival of attention after a trace period and the perception of a click is $d$ (here, $38 \mathrm{msec}$ ). Line ST represents the stimulus train, and line $C T$ the hypothetical central train of survivors. 
train in which all intervals between adjacent pulses are equal. The following train in nonuniform: (1) $/ / / / / /$. So are these, in spite of their periodicities: (2) $/ / / / / / / /$; (3) $/ / 1 / 1 / 1 / 1 / 1 /$.) In general, production by deletion from a uniform stimulus train of a uniform presentation train would demand the periodic loss of at least $50 \%$ of the clicks. In addition, where the stimulus train consists of alternating left and right clicks, and where both ears are to appear in the surviving train, at least two of every three clicks $(67 \%)$ would need to be deleted periodically if a uniform train of survivors is to result.

But subjects do not perceive trains of bilaterally alternating stimuli as nonuniform, and they do perceive them as consisting of left and right stimuli. In our apparent-rate and apparent-numerosity studies, for example, bilateral alternation of pulses results in a perceptually slower or less dense train of pulses than unilaterally presented ones, but the resultant train is perceived as temporally uniform. Likewise, Harvey and Treisman's (1973) subjects, listening to six tone bursts presented in dichotic alternation at $10 / \mathrm{sec}$, claimed that they often heard only three or four of the tones, but that these occurred at regular intervals. This contradiction between the inevitability that deletions of the required order must yield a nonuniform train and the fact of perceived uniformity appears to pose a threat to the proposed models. However, this is so only on the implicit assumption that the resulting nonuniformity can be perceived. The present study examined the perceptibility of objective stimulus nonuniformity in trains of unilateral and bilaterally alternating pulses.

\section{METHOD}

\section{Auditory Click Experiment}

Subjects were 8 female and 7 male students, 17-28 years old. None admitted to any auditory deficit.

Clicks $(58 \mathrm{~dB})$ were produced by impressing $2-\mathrm{msec}, 40-\mathrm{V}$ square pulses across the earphones of a high-impedance headset (Clevite "Brush"). Pulses were generated by two Tektronix Type 161 pulse generators triggered by a Tektronix Type 162 waveform generator. The odd-numbered clicks in a train were produced by the first pulse generator, and the even-numbered ones by the second, which was modified to allow the potentiometer controlling the delay of its pulse to be replaced by an external 10-turn potentiometer. The duration of the earphone's audio response to the pulse was estimated by attaching the earphone to a crystal microphone (Electro-Voice 630) and displaying the microphone response on an oscilloscope at high gain. The major activity was over in about $4 \mathrm{msec}$, and none was discernible after $7 \mathrm{msec}$.

Two standard onset-to-onset interclick intervals (ICIs) were used: 250 and $125 \mathrm{msec}$, producing click rates of $4 / \mathrm{sec}$ and $8 / \mathrm{sec}$, respectively. Each trial consisted of a 4 -sec standard (St) train in which successive clicks were separated by exactly 250 (or 125) msec, a 0.5 -msec silent interval, and a $4-\mathrm{sec}$ comparison (Co) train. In the Co train, even-numbered clicks could be offset in time toward the preceding (negative offset) or succeeding (positive offset) odd-numbered ones, by presenting the external potentiometer; zero offset produced a Co train identical to
St. Figure 3 gives examples of the trains; note the similarity of the offset trains to the periodic nonuniform train (type 2) mentioned earlier. In the terminology provided by Pollack (1967), the standard was a repeated one-interval train, and the comparison a repeated two-interval train with $\mathrm{IPI}_{1}<\mathrm{IPI}_{2}$ (negative offset, $\mathrm{IPI}_{1}>\mathrm{IPI}_{2}$ (positive offset), or IPI $=\mathrm{IPI}_{2}$ (zero off set).

There were two ear conditions, monotic (M) and dichotic (D); in the latter, odd-numbered clicks went to one ear and evennumbered clicks to the other. Eight subjects received trials in the order $5 \mathrm{M}, 10 \mathrm{D}, 5 \mathrm{M}$; seven subjects received trials in the order $5 \mathrm{D}, 10 \mathrm{M}, 5 \mathrm{D}$. Half the $\mathrm{M}$ trials were presented to the left ear, and half to the right; half the $D$ trials began at the left ear, and half at the right.

The subject's task was to judge whether the Co train had a rhythm or beat which was the same as, or different from, that of St. Two abbreviated demonstration trials, $1 \mathrm{M}$ and $1 \mathrm{D}$, were given with the $4 / \mathrm{sec}$ St.

The initial postdemonstration trial for each ear condition began with identical $4 / \mathrm{sec} S t$ and Co trains-i.e., the Co train had zero offset. On succeeding train pairs, positive offset was introduced into the Co train in pair-to-pair stepwise increments of $17.43 \mathrm{msec}$, the trial terminating when the subject gave three successive "different" judgments. The next trial began with a Co offset two to four steps more positive than the terminal setting in the preceding trial, and successive train pairs were given in which the positivity of Co offset was decreased in $17.43-\mathrm{msec}$ steps. The trial continued through zero offset into the negative range, and terminated with the subject's third successive "different." The offset was made $2-4$ steps more negative, and positive-going and negative-going trials then alternated until five complete trials had been given. The initial trialactually a half-trial-was discarded.

The procedure is a variant of the method of just noticeable differences. In a negative-going trial, the point $\mathbf{T}(+)$ was defined as the first transition from "different" to "same," and $T(-)$ as the transition from "same" to the first of three

\section{Offset Zero}

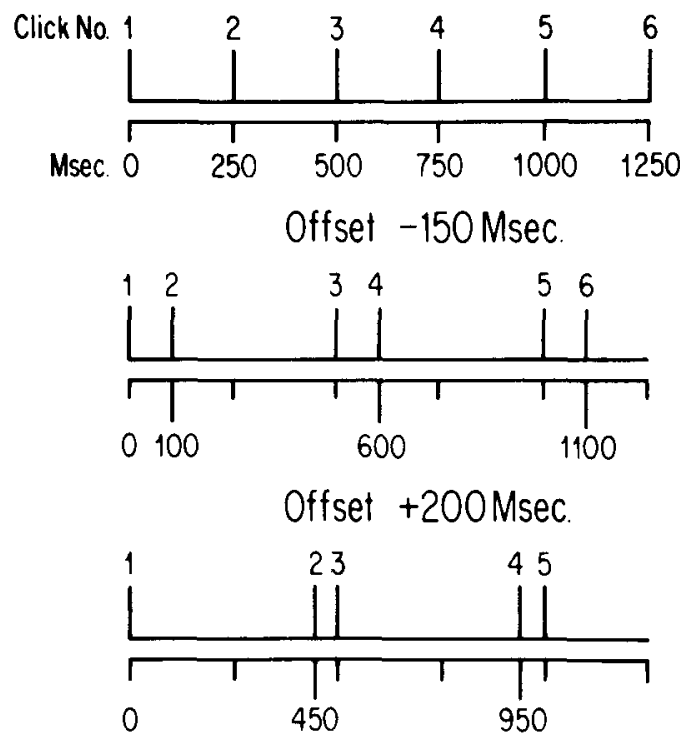

Figure 3. Examples of the first 1,250 msec of comparison (Co) trains, with a standard (St) ICI of 250 msec. A Co train with zero offset consisted of clicks uniformly separated by $250 \mathrm{msec}$, and was therefore identical with the St train. In the train with negative offset, each even-numbered click has been moved 150 msec toward its odd-numbered predecessor; in the train with positive offset, each has been moved 200 msec toward its oddnumbered successor. 
successive "differents." In a positive-going trial, $T(-)$ was the first transition from "different" to "same," and $T(+)$ the transition from "same" to the first of three successive "differents." For each subject under each condition, the difference between the mean $\mathrm{T}(+)$ and the mean $\mathrm{T}(-)$ over the 10 trials was taken as the analog of his interval of uncertainty, which we here call the interval of tolerance (IT), because it represents the band of of fsets acceptable to the subject as not different from the zero offset of St.

The procedure with the $8 / \mathrm{sec}$ standard was similar to that just described, except that the offset step size was $8.80 \mathrm{msec}$. Each subject served under all conditions, receiving the $4 / \mathrm{sec}$ condition first and the $8 / \mathrm{sec}$ condition second.

\section{Somesthetic Tap Experiment}

The subjects were 10 male and 5 female students, 17 to 54 years old; none admitted to somesthetic deficit.

The apparatus was identical to that used in the auditory experiment through the stage eventuating in the outputs of the pulse generators; at that point, the earphones were replaced by transistor circuits which responded to each pulse by impressing $6 \mathrm{~V}$ dc across one of two Ling (Model 102, V-47) vibration generators and a series resistor. Taps were delivered to the palmar surfaces of the middle-finger tips by headless $6 / 32$ bolts inserted into the driving spindles of the generators, the impacts being adjusted by means of the series resistances to be subjectively equal to the experimenters. The initial response of the spindles was an upward movement (toward the skin). The distance between the bolts was $70 \mathrm{~cm}$. White noise masked the sounds made by the generators.

The generators were considerably less deadbeat than the earphones used in the auditory experiment. Sherrick (1964) has reported the response to a $1-\mathrm{msec}$ pulse of a similar device (Goodmans V-47) to be a large positive movement peaking at ca $5 \mathrm{msec}$, a large negative one at ca $9.5 \mathrm{msec}$, and smaller positive movements out to more than $20 \mathrm{msec}$.

Taps were presented unimanually (U) and in bimanual alternation (B). The procedure was identical to that of the auditory experiment, with two exceptions. (a) When a standard intertap interval (ITI) of $125 \mathrm{msec}$ was used with pilot subjects in the $B$ condition, the St and Co trains were usually indistinguishable even at the limiting values of offset (simultaneity of Co taps to the two hands), resulting in inability to obtain meaningful ITs. We therefore used a standard ITI of $167 \mathrm{msec}(6 \mathrm{taps} / \mathrm{sec}$, offset step size $11.72 \mathrm{msec}$ ) for the "fast" somesthetic trains. (b) The St-Co interval was set at $0.8 \mathrm{sec}$, because, with the $1 / 2$-sec interval, pilot subjects complained that they were of ten unable to tell when St ended and Co began.

In analyzing the data, a proportional measure, ITp, was used, where ITp = IT/interval between successive odd-numbered pulses. For comparisons within rates, statistical analyses on the two measures of course yield identical inferences; however, for comparisons between rates, the relative measure is clearly the more appropriate correlate of nonuniformity. Probability levels to be reported for the intramodality results are based on $\mathrm{A}$ (rate) by B (ear or hand condition) by Subject analysis of variance, following Lindquist (1953); in assessing intermodality effects, separate analyses (Lindquist's "type I") were made of the fastrate and the slow-rate data.

The systematic procedure we used to generate nonuniformity in the Co trains did not reproduce the specific type of nonuniformity predicted by either of the two models discussed earlier. However, it has the advantage over quasi-random types of being uniquely specifiable by variation of the temporal position of evennumbered clicks, providing a direct index of the range of nonuniformity perceived as not different from the uniformity of St.

\section{RESULTS}

As is clear from Figure 4, where ITs are shown as bands between two successive odd-numbered stimuli, ITp was wider (a) with bilaterally alternating than with unilateral pulses, (b) with fast than with slow pulses, and (c) with somesthetic than with auditory pulses. The numerical values for mean auditory $\mathrm{ITp} \pm \mathrm{SD}$ (IT in parentheses) were as follows: slow monotic, $.10 \pm .049(51.2 \mathrm{msec})$; slow dichotic, $.19 \pm .068$ (95.0); fast monotic, $.14 \pm .052$ (35.1); fast dichotic, $.30 \pm .092(75.4)$. In somesthesis: slow unimanual, $.14 \pm .060$ (70.9); slow bimanual, $.29 \pm .086$ (143.6); fast unimanual, $.18 \pm .082$ (59.3); fast bimanual, $.44 \pm .179(146.9)$.

In the auditory experiment, the increase of ITp from $M$ to $D$ was significant, as was the increase with rate, ps $<.001$; the effect of ear condition was significantly greater at the fast than at the slow rate, $\mathrm{p}$ (interaction) $<.005$. The ear-condition effect was significant at both rates, ps $<.01$, as was the rate effect in both the $M(p<.05)$ and the $D(p<.01)$ conditions.

Likewise, in somesthesis, both bimanual alternation $(p<.001)$ and increased rate $(p<.005)$ produced significant increases in ITp, the effect of rate being substantially greater in the $B$ than in the $U$ condition $[p$ (interaction $)<.005]$. The hand-condition effect was significant at both rates, ps $<.01$. The increase in ITp with increased rate was not significant in the $U$ condition, $p>.20$; in $B, p<.01$.

Comparison of Panels $A$ and $C$ in Figure 4 reveals that, with stimuli presented at the rate of $4 / \mathrm{sec}$, the somesthetic ITps were wider than the auditory, $\mathrm{p}<.01$, the modality effect being greater in the twosource $(D, B)$ than in the one-source $(M, U)$ conditions, $\mathrm{p}$ (interaction) $<.005$. The increase from audi-

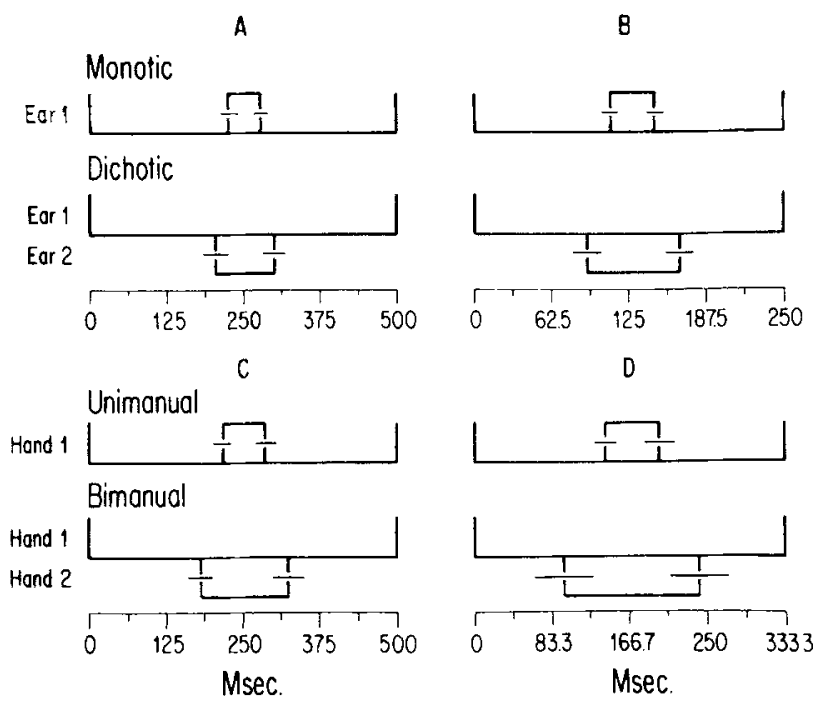

Figure 4. Mean intervals of tolerance (rectangles), and \pm 1 standard deviation of the upper and lower thresholds. The upwardgoing lines represent successive odd-numbered clicks. Trains were 4 sec long; only one cycle is pictured for each condition. Panel A: Standard interclick interval $=250 \mathrm{msec}$. Panel $\mathrm{B}$ : St $\mathrm{ICI}=$ 125 msec. Panel $C$ : Standard intertap interval $=250 \mathrm{msec}$. Panel D: St. ITI $=167 \mathrm{msec}$. 
tion to somesthesis failed of significance in the onesource condition, . $05<\mathrm{p}<.10$; in the two-source condition, $\mathrm{p}<.01$.

Panels $B$ and $D$, representing the results with the faster rates, show a pattern similar to that of Panels A and $C$. The somesthetic ITps were wider than the auditory ones, $p<.025$, even though the faster somesthetic rate was slower than the faster auditory rate. The widening effect of alternation on ITp was again greater in somesthesis than in audition $p($ interaction) $<.025$. The difference in ITp between somesthesis and audition in the one-source condition did not reach significance, $.10<p<.20$; in the twosource condition, $p<.02$. Because the somesthetic fast rate was slower than the auditory, these last two comparisons doubtless underestimate the modality effect.

\section{DISCUSSION}

Our results fit in well with some of those obtained by Pollack $(1968 \mathrm{a}, \mathrm{b})$ in a series of parametric studies on perception of auditory temporal irregularity. Nonuniform ("jittered") diotic trains were produced by randomly increasing or decreasing, by an amount $\mathbf{J}$, each interpulse interval of initially uniform (oneinterval repeated) trains, and the threshold $\mathrm{J}$ required for discrimination of the jittered from the unjittered trains was determined. At the slowest rate he used, ca 30 pulses/sec, Pollack (1968a) obtained proportional jitter thresholds, J/IPI, ranging between ca .03 and .15. Pollack's experiment differed from ours in other ways than repetition rate and rule for assigning offsets; e.g., he used a four-interval forced-choice procedure, and most of his subjects had had musical training. Considering these differences, the sizes of the monotic ITps obtained here $(.10, .14)$ seem reasonable. The monotic ITs we obtained were likewise of the same order of magnitude-10s of milliseconds-as those might have been expected, with the rates we used, on the basis of earlier results of Pollack's (1967, Figure 9) with two-interval nonrepeated (three-pulse) diotic trains in well-practiced, musically trained subjects. With respect to the effect of dichotic presentation, the present results are consistent with Pollack's (1968b) finding that jitter thresholds were consistently higher when each pulse was randomly delivered to one or the other ear than when the trains were presented diotically; that is, that jitter was systematically less discriminable in dichotic trains than in trains coming from a single (apparent) source. The ratios of the dichotic to the diotic jitter thresholds declined from between ca 150 and $18: 1$ to between ca 8 and $2: 1$ as repetition rate declined from 4,000 to 30 pulses/sec; the corresponding ratios in the present work were close to $2: 1$ (Results, above, and Figure 4).
Our results indicate that failure per se to perceive temporal nonuniformity in trains of rapidlyalternating stimuli is not yet lethal to explanatory models, such as those exemplified in Figures 1 and 2, which entail the implication that the surviving members of an alternating train will be temporally nonuniform: substantial amounts of input nonuniformity go unnoticed as such, even when the observer makes direct comparisons on each trial between the uniform and nonuniform trains. ${ }^{3}$

Nevertheless, if deletion models are to remain viable, they would seem to need provision for an additional process of temporal respacing in the resultant central train. Any single dropout from an $8 / \mathrm{sec}$ train of alternating clicks, for example, would leave a gap of $250 \mathrm{msec}$, and dropout of two successive clicks a gap of $375 \mathrm{msec}$. Although the stimulus trains in the present investigation were not generated by such dropouts, the fact that the threshold offset for the $8 / \mathrm{sec}$ alternating clicks was less than $40 \mathrm{msec}$ (IT/2) certainly suggests that gaps of the order of several hundred milliseconds would result in perceived nonuniformity. Respacing is built into the switching model (compare ST and CT in Figure 2); but the probabilistic inhibition model would need additional assumptions.

In discussing their experiments on attentionshifting in a tone-monitoring task, Harvey and Treisman (1973) have proposed a mechanism which may provide an account for several phenomena of experiments with bilaterality alternating stimuli. Assume first that the elements in a train queue up in a short-term buffer, where they are subject to dropout because of decay with time before being processed by a perceptual/attentional mechanism. Assume further that alternating stimuli require additional processing time for spatial-location analysis. Finally, assume that "the temporal arrangement perceived depends on the [uniform] output times of the perceptual mechanism rather than on the ... arrival times of the subset of stimuli that it is able to accept" (p. 55, italics in original). These assumptions produce the following consequences. (a) Because the processing time for any alternating stimulus is extended, subsequent stimuli must wait longer in the queue, and are therefore more subject to decay. This would account for decreased apparent numerosity of alternating stimuli. (b) The temporal spacing of the output will be wider than that of the presentation train-i.e., there will be an apparent slowing of alternating stimuli. (c) Although the deletions resulting from decay may leave a nonuniform set of survivors, the uniformity of output of the perceptual mechanism would impose itself on the queued stimuli; the imposed uniformity would fail-i.e., nonuniformity would be perceived-only if there were no elements in the buffer. The failure to per- 
ceive the nonuniformity of both objectively nonuniform stimulus trains and the hypothetical central trains made nonuniform by deletions from uniform stimulus trains are thus both accounted for.

One problem with this account, which it shares with the attention-shifting model of Figure 2, and which appears to necessitate additional assumptions, is that it implies an absolute ceiling to the output rate of the perceptual mechanism, no matter by how much the input rate exceeds the output rate. But this is counter to the finding that as alternatingstimulus rates increase beyond those that first produce perceptual slowing or decreased numerosity, the perceived rate and numerosity also continue to increase (e.g., Axelrod et al., 1968; Guzy \& Axelrod, 1972). Guzy and Axelrod (1972) have suggested that it may be useful to think of interaural attentionshifting as analogous to a motor response. On such an analogy, one could imagine that the shifting mechanism might be driven to operate more rapidly by more rapid stimuli. Another problem is that subjects do perceive nonuniform alternating trains as such when the nonuniformity exceeds the threshold-e.g., when offset was greater than IT/ 2 in the present experiments. The Harvey/Treisman hypothesis does not appear able to account for this veridical perception in the supraliminal case.

Our own introspections, and the spontaneous reports of some of our subjects, suggest the possibility of an altogether different variety of account of degradations of the processing of alternating stimuli. When one hears or feels rapid alternating trains, the experience is often not one of a single integrated train. Rather, one can experience two parallel but independent trains, a left and a right; cross-train judgments concerning temporal relations among the stimuli can therefore be remarkably difficult to make. This is reminiscent of Bregman and Campbell's (1971) description of the preattentive process of "primary auditory stream segregation" by pitch, as a result of which ". ... Ss report in a stream-bystream order, cannot identify sequences that cross the organizational streams, and have no clear idea of temporal relations between the two streams" (p. 249). ${ }^{4}$ We have elsewhere (Axelrod \& Nakao, 1974) proposed extension of the stream-segregation concept to segregation-by-locus in audition and somesthesis, and recent work by Bregman and Achim (1973) has demonstrated stream segregation in vision, with spatial location functioning analogously to auditory pitch. [Indeed, the concept might fruitfully be further generalized to cover all cases of alternation between heterogeneous stimuli, including modality alternation (Holden, 1973).] On this account, the difficulty in interdigitating alternating stimuli would arise not in shifting repetitively between successive stimuli, but in shifting between the resultant streams. Our findings that ITp increased with stimulus rate is consistent with Bregman and Achim's (1973) and Bregman and Dannenbring's (1973) reports that stream segregation is likewise a function of rate. However, stream segregation does not appear able to account for deletion of the required order, i.e., $20 \%-40 \%$.

Axelrod and Nakao (1974) proposed that there might be a single central mechanism which limits the processing of alternating stimuli in either modality. The modality effects in the present study are not necessarily damaging to that proposal, as they may be accounted for by differences in more or less peripheral, modality-specific processes. (a) The somesthetic transducer we used rang considerably longer than the earphone (Method, above), so that the duration of the somesthetic pulse was longer than that of the click. (b) The somesthetic system is more sluggish than the auditory; see, e.g., von Békésy's determinations $(1960$, pp. 358,536$)$ that the interval between the onset of a vibratory burst and its maximum perceived intensity is ca $180 \mathrm{msec}$ in audition and ca $1,200 \mathrm{msec}$ in touch. (c) Gescheider (1966) has found liminal $\Delta t$ for the resolution of successiveness of paired pulses to be much longer for touch (10-12.5 msec) than for hearing $(1.6-1.8 \mathrm{msec})$. Such basic sensory differences in temporal response would be expected to produce more uncertainty about temporal sequences in somesthesis than in audition, and so increase the size of IT.

\section{REFERENCES}

AXElroD, S., \& GuzY, L. T. Underestimation of dichotic click rates: Results using methods of absolute estimation and constant stimuli. Psychonomic Science, 1968, 12, 133-134.

Axelrod, S., Guzy, L. T., \& Diamond, I. T. Perceived rate of monotic and dichotically alternating clicks. Journal of the Acoustical Society of America, 1968, 43, 51-55.

Axelrod, S. \& NAKAO, M. Apparent slowing of bimanually alternating pulse trains. Journal of Experimental Psychology, 1974, 102. 164-166.

Bregman. A. S.. \& Achim, A. Visual stream segregation. Perception \& Psychophysics, 1973, 13, 451-454.

Bregman, A. S., \& Campbell, J. Primary auditory stream segregation and perception of order in rapid sequences of tones. Journal of Experimental Psychology, 1971, 89, 244-249.

Bregman, A. S., \& Dannenbring, G. L. The effect of continuity on auditory stream segregation. Perception \& Psychophysics. $1973,13,308-312$.

GesCheIDER, G. A. Resolving of successive clicks by the ears and the skin. Journal of Experimental Psychology, 1966,-71. 378-381.

Guzy, L. T., \& Axelrod, S. Interaural attention shifting as response. Journal of Experimental Psychology, 1972, 95, 290-294.

Harvey, N., \& Treisman, A. M. Switching attention between the ears to monitor tones. Perception \& Psychophysics, 1973, 14, 51.59.

Holden, E. A., JR. Effects of stimulus alternation on sequential information processing by retarded and nonretarded subjects. Journal of Experimental Psychology, 1973, 101, 262-268.

Huggins. A. W. F. On perceptual integration of dichotically alternating pulse trains. Journal of the Acoustical Society of America, 1974, 56, 939-943. 
Jenkins, R. A. Perception of pitch, timbre, and loudness. Journal of the Acoustical Society of America, 1961, 33, 1550-1557.

Kahneman, D. Remarks on attention control. Acta Psychologica, 1970, 33, 118-131.

LINDQUist, E. F. Design and analysis of experiments in psychology and education. Boston: Houghton Mifflin, 1953.

McClellan, M. E., \& Small, A. M., JR. Pitch perception of pulse pairs with random repetition rate. Joumal of the Acoustical Society of America, 197, 41, 690-699.

Pollack, E. Asynchrony: The perception of temporal gaps within periodic auditory pulse patterns. Joumal of the Acoustical Society of America, 1967, 42, 1335-1340.

Pollack. I. Detection and relative discrimination of auditory "jitter." Joumal of the A coustical Society of America, 1968, 43, 308-315. (a)

Pollack, l. Can the binaural system preserve temporal information for jitter? Joumal of the Acoustical Society of America, $1968,44,968-972$. (b)

SHERRICK. C. E. Effects of double simultaneous stimulation of the skin. American Journal of Psychology, 1964, 77, 42-53.

Small, A. M., JR., \& MCClellan, M. E. Pitch associated with time delay between two pulse trains. Joumal of the Acoustical Society of America, 1963, 35, 1246-1255.

Thurlow, W. R.. \& Hartman, T. F. The "missing fundamental" and related pitch effects. Perceptual and Motor Skills, 1959, 9, 315-324.

Treisman, A. M. Shifting attention between the ears. Quarterly Journal of Experimental Psychology, 1971, 23, 157-167.

voN BeKEsY, G. Experiments in hearing. New York: McGrawHill, 1960.

W ALLEY, R. E., \& WEIDEN, T. D. Lateral inhibition and cognitive masking: A neuropsychological theory of attention. Psychological Review, 1973, 80, 284-302.

\section{NOTES}

1. An anonymous reviewer has pointed out that the validity of concluding that $30 \%-40 \%$ of alternating clicks are lost from the fact that alternating trains are perceived as $60 \%-70 \%$ as fast as otherwise identical monotic trains depends on linearity of the function relating estimated (perceived) monotic numerosity to actual monotic numerosity. If (a) the function is in fact negatively accelerated, and if (b) the curvature is sufficiently great, then (c) attention to one ear at a time in the alternating condition at $\mathrm{x} / \mathrm{sec}$ might generate an estimate appropriate to $(\mathrm{x} / \mathrm{sec}) / 2$ in the monotic condition. Guzy and Axelrod (1972) obtained numerosity estimates for trains of 2-20 clicks presented at several rates. Plots of estimated vs. presented number (monotic) depart only slightly from linearity (though in the direction of negative acceleration)-so slightly as to make condition $b$ and thus also inference $c$ clearly invalid. The same conclusion can be derived from the data on absolute estimates of rate reported by Axelrod and Guzy (1968).

2. Huggins (1974) presented a standard dichotically alternating train and a binaural one whose rate the subject could vary continually; the subject could also switch back and forth at will between the standard and variable until he was satisfied with the match. At slow rates, two of the three intensively studied subjects whose data Huggins presents adjusted the binaural train to the veridical dichotic rate, the matches then dropping rather abruptly to about $50 \%$ of the alternating rate-i.e., to the rate in one ear-at dichotic rates around $10-20 / \mathrm{sec}$. (When required to adjust the binaural rate to that of one ear in the dichotic train, all three subjects' matches were very close to veridical.) The third subject never reached the $50 \%$ level at the fast rates when matching the binaural to the dichotic rate, and showed significant, though small, deviations from his rate-in-oneear matches. Both Huggins' procedures and his results were thus substantially different from those we have reported earlier. In the present study, we employed our usual procedure of presenting discrete alternating and unilateral trains, requiring subjects to make verbal judgments about them.

3. A.M. Small, Jr., and his colleagues, working with selected well-practiced subjects, have shown that monotic pulse trains formally similar to our finite-offset Co trains can result in perception of a pitch which the subjects can match rather precisely with a pure tone. Most commonly, the match is a tone whose period approximates the shorter of the two interpulse intervals of a triplet (the fundamental "time-separation pitch," TSP); much less often, it is an octave of that tone ("octave error"). It is unlikely that TSP had any important effect on our results: (a) TSP is particularly difficult to hear under the constantoffset condition which obtained in each Co train; indeed, Jenkins (1961) reported inability to demonstrate TSP with such trains. [In Small's experiments, special efforts were often made to help the subject "hear out" the TSP (McClellan \& Small, 1967, p. 694; Small \& McClellan, 1963, pp. 1247-8). Thurlow and Hartman (1959) swept the predicted TSP briefly from $250 \mathrm{~Hz}$ (the target pitch) down to $200 \mathrm{~Hz}$ and then back up again to a steady $250 \mathrm{~Hz}$, to call the subjects' attention to the TSP; even so, of 35 subjects, all with some musical training, only 5 adjusted a matching oscillator to within $10 \%$ of the target, and 6 to within $10 \%$ of one octave of the target.] (b) Our subjects-unselected except as having presumptively normal hearing-were specifically instructed to attend to the rhythmic attributes of the trains. (c) Of the 600 monotic transition points in the auditory experiment, not one occurred at an offset large enough to give rise to a fundamental TSP within the audible range $(20 \mathrm{~Hz}$ or higher). The closest approach, in 4 of the 300 transitions at the first rate, correspond to a time separation of ca $77 \mathrm{msec}(\mathrm{ca} 13 \mathrm{~Hz}$ ), three offset-increment steps longer than the $50-\mathrm{msec}$ separation appropriate to a $20-\mathrm{Hz}$ TSP. So far as we know, TSP has not been reported for time separation $>20 \mathrm{msec}(50 \mathrm{~Hz})$.

4. A related difficulty in making temporal judgments about dichotically alternating pulses is reported by Harvey and Treisman (1973), who presented six-element trains monotically and in dichotic alternation; tones appeared in $3,4,5$, or 6 of the 100 -msec intervals, the remaining elements consisting of silence. Subjects were remarkably poorer in specifying the temporal positions of the filled and empty intervals in the dichotic trains than in the monotic ones.

(Received for publication July 26, 1976; accepted for publication August 5, 1976.) 